\title{
Inverse scattering analysis for an elastic half space based on a fast volume integral equation method
}

\author{
T. Touhei, T. Takagishi, Y. Wajima \& K. Kuranami \\ Department of Civil Engineering, Tokyo University of Science, Japan
}

\begin{abstract}
A method for the inverse scattering analysis is presented for an elastic half space. The volume integral equation method is introduced here to reconstruct fluctuation of the medium from scattered waves observed at the free surface. The Born approximation is applied to the volume integral equation to formulate the equation for the inverse scattering analysis. The equation is solved by means of the Krylov subspace iteration method and the fast generalized Fourier transform. Several numerical calculations are carried out to investigate the solutions of the inverse scattering analysis.

Keywords: inverse scattering problem, volume integral equation, elastic half space, fast generalized Fourier transform, Krylov subspace iteration method, Born approximation.
\end{abstract}

\section{Introduction}

The analysis of scattered waves is an important issue in fields such as earthquake engineering, non-destructive testing and identification of energy resources. The integral equation methods have been efficient tools for both the forward and inverse scattering analysis. For example, Colton and Kress (1998) presented a survey of a vast number of articles on forward and inverse scattering analyses [1]. Among the integral equation methods, the volume integral equation known as the Lippmann-Schwinger equation has an advantage in that it presents a mathematical relationship between the fluctuation of the medium and scattered wave field. As a result, a number of examples of applications of the volume integral equation are increasing recently in spite of its deficiency, that is the requirement of a huge scale 
and dense matrix for numerical analyses. Several methods for the volume integral equation presented recently are aimed to resolve the deficiency of the volume integral equation (for example, [2-4]). Even in this situation, application of the volume integral equation to inverse scattering analysis of an elastic half space is still a task for the future.

In this article, a method for inverse scattering analysis is presented for an elastic half space by means of the volume integral equation. The inverse scattering analysis here is to reconstruct fluctuation of the medium from the scattered waves observed at the free surface. The formulation is carried out by applying the Born approximation to the volume integral equation. As a result, the equation is obtained for the reconstruction of fluctuation of the medium from scattered wave field. The introduction of the fast generalized Fourier transform [5] and the Krylov subspace iteration method [6] enables us to solve the equation. Several numerical examples by means of the proposed method are presented in this article.

\section{Theoretical formulation}

\subsection{Problem dealt in this article}

Consider a scattering problem shown in Fig. 1, in which a point source is applied to a free surface of an elastic half space. The wave field has a localized fluctuation and scattered waves are caused due to an interaction between the incident waves and fluctuation of the medium. The problem of this article is to reconstruct the fluctuation by means of scattered waves. We assume that the back ground structure of the wave field is already provided for the analysis.

According to Fig. 1, the set of the points for grids to observe the scattered waves is denoted by $\Gamma_{O}$ and those to reconstruct the fluctuation is denoted by $\Gamma_{R}$. To set up $\Gamma_{R}$, the properties of the waves due to a point source is used here. As shown in later, the waves due to a point source propagating to downward direction are found

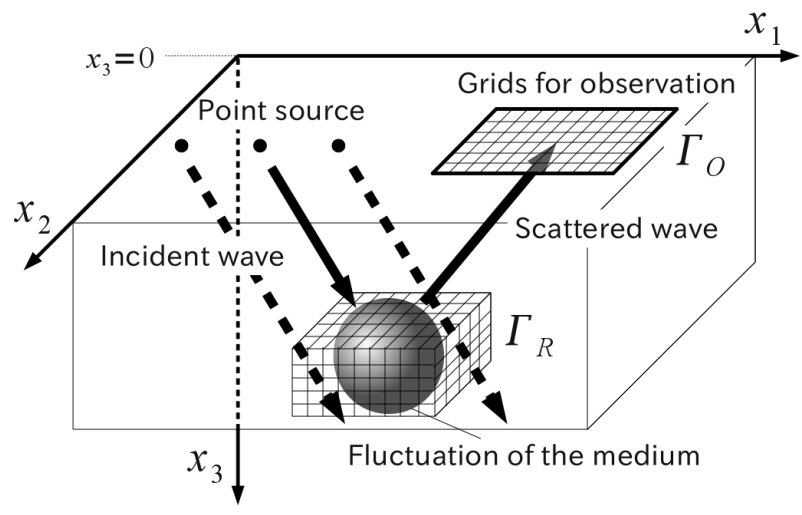

Figure 1: Concept of the scattering problem. 
to exhibit a strong directionality. Therefore, by means of the position of the point source and the intensity of the scattered waves observed at the free surface, it is possible to set up grids for $\Gamma_{R}$. This concept is also described in Fig. 1.

The fluctuation of the medium is defined by the Lamé constants and the mass density such that

$$
\begin{aligned}
& \lambda(x)=\lambda_{0}+\tilde{\lambda}(x) \\
& \mu(x)=\mu_{0}+\tilde{\mu}(x) \\
& \rho(x)=\rho_{0}+\tilde{\rho}(x), \quad\left(x \in \mathbb{R}_{+}^{3}\right)
\end{aligned}
$$

where $x \in \mathbb{R}_{+}^{3}$ is the spatial point, $\lambda_{0}, \mu_{0}$ and $\rho_{0}$ are the background constants of the Lamé and mass density and $\tilde{\lambda}, \tilde{\mu}$ and $\tilde{\rho}$ are their fluctuations. Note that the components of $x \in \mathbb{R}_{+}^{3}$ are expressed by

$$
x=\left(x_{1}, x_{2}, x_{3}\right) \in \mathbb{R}_{+}^{3}
$$

where $x_{3}$ is the vertical coordinate with the positive direction downward and the free boundary is defined by $x_{3}=0$.

The inverse scattering analysis dealt in this article can now be stated as follows:

Definition 1 The inverse scattering analysis is to reconstruct the fluctuation of the medium $\tilde{\lambda}, \tilde{\mu}$ and $\tilde{\rho}$ at $\Gamma_{R}$ from information about the scattered waves observed at the grids $\Gamma_{O}$, the background structure of the medium and the incident wave field.

\subsection{Volume integral equation}

The volume integral equation for the scattering problem shown in Fig. 1 is described as [4]

$$
\begin{aligned}
u_{i}(x)= & -\int_{\mathbb{R}_{+}^{3}} g_{i j}(x, y) N_{j k}(y) u_{k}^{(I)}\left(y, x_{s}\right) d y \\
& -\int_{\mathbb{R}_{+}^{3}} g_{i j}(x, y) N_{j k}(y) u_{k}(y) d y, \quad\left(x \in \mathbb{R}_{+}^{3}\right)
\end{aligned}
$$

where $u_{i}$ is the scattered wave field, $g_{i j}$ is the Green's function for an elastic half space due to the background structure, $N_{i j}$ is the differential operator constructed by the fluctuations of the medium, $u_{i}^{(I)}$ is the incident wave field caused by a point source and $x_{s}$ is the spatial point where the point source is applied. The subscript indices $i, j$ and $k$ for Eq. (3) denotes the components of the vectors or tensors to which the summation convention is applied.

The Green's function for an elastic half space used for the integral equation is defined by

$$
\left(\left(\lambda_{0}+\mu_{0}\right) \partial_{i} \partial_{j}+\delta_{i j} \mu_{0} \partial_{l} \partial_{l}+\delta_{i j} \rho_{0} \omega^{2}\right) g_{j k}(x, y)=-\delta_{i k} \delta(x-y)
$$


114 Boundary Elements and Other Mesh Reduction Methods XXXII

$$
P_{i j} g_{j k}(x, y)=0 \text { at } x_{3}=0
$$

where $\partial$ is the partial differential operator, $\delta_{i j}$ is the Kronecker delta, $\omega$ is the circular frequency, and $P_{i j}$ is the differential operator expressed as

$$
\left[P_{i j}\right]=\left[\begin{array}{ccc}
\mu_{0} \partial_{3} & 0 & \mu_{0} \partial_{1} \\
0 & \mu_{0} \partial_{3} & \mu_{0} \partial_{2} \\
\lambda_{0} \partial_{1} & \lambda_{0} \partial_{2} & \left(\lambda_{0}+2 \mu_{0}\right) \partial_{3}
\end{array}\right]
$$

For numerical analysis here, the Green's function defined by Eqs. (4) and (5) is constructed by the fast generalized Fourier transform. By means of the Green's function, the incident wave field is calculated such that

$$
u_{k}^{(I)}\left(x, x_{s}\right)=g_{k l}\left(x, x_{s}\right) \beta_{l}
$$

where $\beta_{l}$ is the force vector of the point source. In addition, the explicit form of the differential operator $N_{i j}$ in Eq. (3) is expressed by

$$
\begin{aligned}
N_{i j}(x)= & -(\tilde{\lambda}(x)+\tilde{\mu}(x)) \partial_{i} \partial_{j}-\delta_{i j} \tilde{\mu}(x) \partial_{k} \partial_{k} \\
& -\partial_{i} \tilde{\lambda}(x) \partial_{j}-\delta_{i j} \partial_{k} \tilde{\mu}(x) \partial_{k}-\partial_{j} \tilde{\mu}(x) \partial_{i}-\delta_{i j} \tilde{\rho}(x) \omega^{2}
\end{aligned}
$$

\subsection{Method for the inverse scattering analysis}

At this stage, let us modify the volume integral equation into that represents the relationship between the observed scattered waves at $\Gamma_{O}$ and fluctuation of the medium at $\Gamma_{R}$. Application of the Born approximation to the volume integral equation shown in Eq. (3) yields

$$
u_{i}(x)=-\int_{\mathbb{R}_{+}^{3}} g_{i j}(x, y) N_{j k}(y) u_{k}^{(I)}\left(y, x_{s}\right) d y
$$

The right side of Eq. (9) can be modified so that the unknown quantities are the fluctuation of the medium. Let $q_{k}$ be the component of the states vector with respect to the fluctuation of the medium such that

$$
\left(q_{k}\right)=\left(\begin{array}{lll}
\tilde{\lambda} & \tilde{\mu} & \tilde{\rho}
\end{array}\right)^{T}
$$

Then, Eq. (9) becomes as follows:

$$
u_{i}(x)=\int_{\mathbb{R}_{+}^{3}} g_{i j}(x, y) M_{j k}\left(y, x_{s}\right) q_{k}(y) d y
$$

where $M_{i j}$ is the operator including the effects of the incident wave field, which is defined by

$$
N_{j k}(y) u_{k}^{(I)}\left(y, x_{s}\right)=-M_{j k}\left(y, x_{s}\right) q_{k}(y)
$$


The explicit form of $M_{j k}$ is expressed as

$$
\left[M_{j k}\right]=\left[\begin{array}{ccc}
\partial_{1} E_{l l}+E_{l l} \partial_{1} & \partial_{1} E_{l l}+\eta_{1}+2 E_{1 l} \partial_{l} & \omega^{2} u_{1}^{(I)} \\
\partial_{2} E_{l l}+E_{l l} \partial_{2} & \partial_{2} E_{l l}+\eta_{2}+2 E_{2 l} \partial_{l} & \omega^{2} u_{2}^{(I)} \\
\partial_{3} E_{l l}+E_{l l} \partial_{3} & \partial_{3} E_{l l}+\eta_{3}+2 E_{3 l} \partial_{l} & \omega^{2} u_{3}^{(I)}
\end{array}\right]
$$

where $E$ and $\eta$ in Eq. (13) are defined by

$$
\begin{aligned}
E_{i j} & =(1 / 2)\left(\partial_{i} u_{j}^{(I)}+\partial_{j} u_{i}^{(I)}\right) \\
E_{l l} & =E_{11}+E_{22}+E_{33} \\
\eta_{j} & =\left(\partial_{1}^{2}+\partial_{2}^{2}+\partial_{3}^{2}\right) u_{j}^{(I)}
\end{aligned}
$$

Note that the summation convention is applied to the subscript index $l$ in Eq. (13).

At this stage, the generalized Fourier and its inverse transforms are applied to Eq. (11). In addition, assume that

$$
\begin{aligned}
q_{k}(y) & =0, \quad\left(y \in \mathbb{R}_{+}^{3} \backslash \Gamma_{R}\right) \\
x & \in \Gamma_{O}
\end{aligned}
$$

for Eq. (11). Then, Eq. (11) becomes the equation for the inverse scattering analysis, which is in the following form:

$$
u_{i}(x)=\mathscr{U}_{i j}^{-1} \hat{h}(\xi) \mathscr{U}_{j k} M_{k l}\left(y, x_{s}\right) q_{l}(y), \quad\left(x \in \Gamma_{O}\right),
$$

where $\mathscr{U}$ and $\mathscr{U}^{-1}$ are the operators for the generalized Fourier and its inverse transforms, respectively, and $\hat{h}$ is the function related to the generalized Fourier transform of the Green's function expressed by

$$
\hat{h}(\xi)=\frac{1}{\mu_{0} \xi_{3}^{2}-\rho_{0} \omega^{2}+i \epsilon}
$$

where $\epsilon$ is an infinitesimally small positive number and $\xi$ is the wavenumber vector having components

$$
\xi=\left(\xi_{1}, \xi_{2}, \xi_{3}\right) \in \sigma_{p} \cup \sigma_{c} \subset \mathbb{R}_{+}^{3}
$$

Note that $\sigma_{p}$ and $\sigma_{c}$ are the set of the wavenumber for the Rayleigh and the body waves, respectively. The detail of the discussions for these sets are given in the article [4].

Now, we can solve Eq. (17) by means of the fast generalized Fourier transform and the Krylov subspace iteration method. Note that the present method does not require the derivation of a coefficient matrix. During the iterative process, only the discretized transforms are repeatedly used for the analysis. 


\section{Numerical examples}

\subsection{Analyzed target model and incident wave}

Preliminary to carry out an inverse scattering analysis, let us set up a target model for the analysis. The back ground Lamé constants and mass density for the model are $\lambda_{0}=4.0 \mathrm{GPa}, \mu_{0}=2.0 \mathrm{GPa}$ and $\rho_{0}=2.0 \mathrm{~g} / \mathrm{cm}^{3}$, respectively. Namely, the P wave velocity for the back ground structure of the wave field is $2 \mathrm{~km} / \mathrm{s}$ and the $\mathrm{S}$ wave velocity for that is $1 \mathrm{~km} / \mathrm{s}$. The fluctuation of the medium defined by $\tilde{\lambda}$ and $\tilde{\mu}$ is shown in Fig. 2(a). The fluctuation of the Lamé constants are in the form of a cube whose amplitudes are $0.2 \mathrm{GPa}$. In addition, the fluctuated area is embedded at the depth of $3.0 \mathrm{~km}$ from the free surface.

An incident wave field used for the analysis is shown in Fig. 2(b), that is constructed by the fast generalized Fourier transform. For the discretization of the generalized Fourier transform, the intervals of the grids in the space domain and the number of grids for the $j$-th coordinate $(j=1,2,3)$ are set by $\Delta x_{j}=0.25 \mathrm{~km}$ and $N_{j}=256$, respectively. The amplitude of the point source for the incident wave field is $1.0 \times 10^{10} \mathrm{~N}$, whose direction is in vertical, and the excitation frequency is $1.0 \mathrm{~Hz}$. As can be seen in Fig. 2(b), the high displacement amplitudes areas can be seen along the free surface as well as downward directions from the free surface. These areas are corresponding to the Rayleigh and the body waves, respectively. The body waves propagating to downward direction are found to show the strong directionality and expected to interact with the fluctuation of the medium.

Figures 3(a) and (b) show the propagation of scattered waves at the free surface and the amplitude of the scattered waves in $x_{1}-x_{3}$ plane, respectively. According to Fig. 3(a), the displacement of the scattered waves are larger in the forward region of the fluctuation of the medium. In addition, some spots showing larger

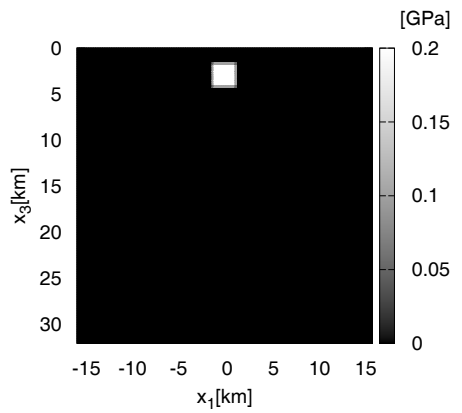

(a) fluctuations of $\tilde{\lambda}$ and $\tilde{\mu}$

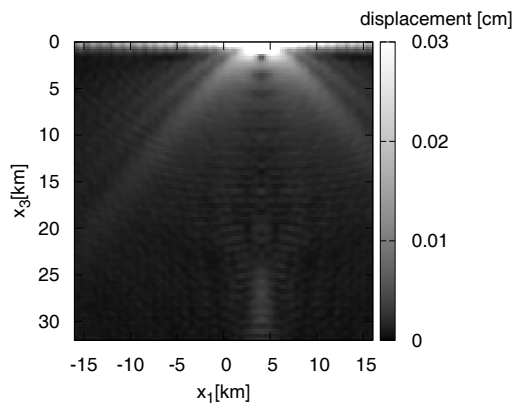

(b) incident wave field

Figure 2: Fluctuation of the medium and incident wave field. 


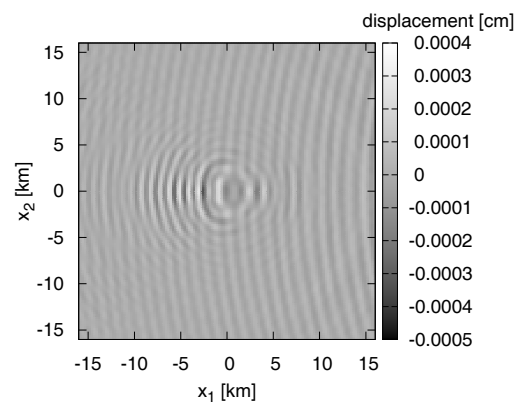

(a) displacement at the free surface

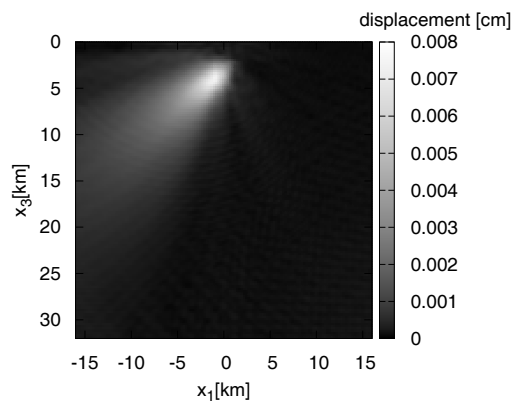

(b) displacement amplitudes in the vertical plane

Figure 3: The result of forward scattering analysis. The displacement of the scattered waves are shown.

displacement can be seen in the forward region of the fluctuation. These spots are caused due to the reflection of the body waves from the fluctuation of the medium, from which the directionality of the body waves can be recognized. It is also found from Fig. 3(b) that the outstanding of the displacement amplitudes in the forward direction also can be seen. The displacement amplitudes in the vertical plane is found to be much larger than that in the free surface. This indicates that the refection of the body waves from the fluctuated area to the free surface is not very large when compared to the scattered waves propagating to downward direction from the fluctuation of the medium.

\subsection{Examples of the inverse scattering analysis}

At this stage, let us reconstruct the fluctuation of the medium. Figures 4(a) and (b) show a range of data sampling of scattered waves at the free surface and the results of the reconstruction of $\tilde{\mu}$ at the depth of $3 \mathrm{~km}$ from the surface. Likewise, Figs 5(a) and (b) also show a range of data sampling and the results of the reconstruction of $\tilde{\mu}$ at the depth of $3 \mathrm{~km}$. The differences of the data samplings between Figs. 4 and 5 are that Fig. 4 takes the data of scattered waves of the forward direction while Fig. 5 takes those of the backward direction.

It is found from Fig. 4(b) that the highest amplitude of the estimated fluctuation is $2.0 \mathrm{GPa}$. Since the target of the fluctuation was $0.2 \mathrm{GPa}$ as shown in Fig. 2(a), the estimation of the fluctuation is higher here. According to the estimation shown in Fig. 5(b), the highest amplitude is $5.0 \mathrm{GPa}$. In this case, the estimated fluctuation is much higher than that shown in Fig. 4. Namely, the discrepancy between the target and estimation of the fluctuation increases in Fig. 5. The reason for this is clearly due to the properties of the sampled scattered waves, which convey the information for the fluctuation of the medium. These results indicate that sampling 


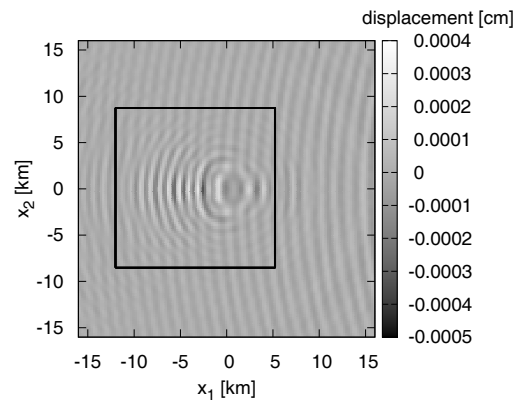

(a) range of the data sampling

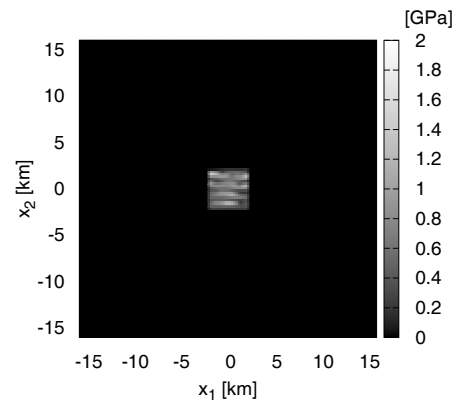

(b) reconstruction of $\tilde{\mu}$ at the depth of $3 \mathrm{~km}$

Figure 4: The result of inverse scattering analysis.

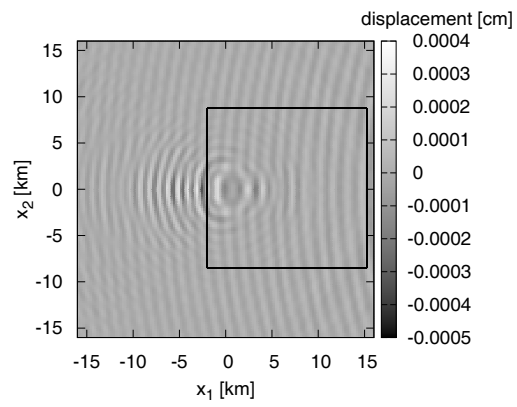

(a) range of the data sampling

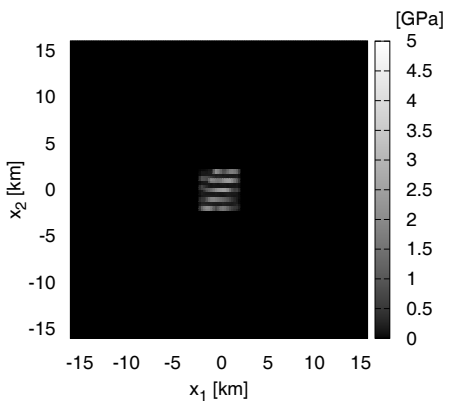

(b) reconstruction of $\tilde{\mu}$ at the depth of $3 \mathrm{~km}$

Figure 5: The result of inverse scattering analysis.

of the scattered waves should be in the forward direction of the fluctuation. Note that the accuracy of the reconstructed fluctuation here has to be improved for the future. We have to examine the effects of the excitation frequency, the intervals of the grids, number of data sampling and among others.

The numerical calculations were carried out by a computer with an AMD Opteron $2.4 \mathrm{GHz}$ processor. The CPU time needed for the present example based on the Bi-CGSTAB method was around $150 \mathrm{~min}$, in which the number of the iterations was 10 times to obtain the results. 


\section{Conclusion}

This article presented a method for inverse scattering analysis based on the volume integral equation method. The Born approximation was applied to the volume integral equation to obtain the equation for the inverse scattering analysis, which connected the scattered waves observed at the free surface and fluctuation of the medium. The equation was solved by the fast generalized Fourier transform and the Krylov subspace iteration method. Several numerical calculations were carried out to examine the solutions of the inverse scattering analysis. According to the numerical calculations, the amplitude of the estimated fluctuations of the medium were higher than that of the target. Properties of the sampled scattered waves were found to affect significantly to the results of the reconstruction of the fluctuation. Improvements of the accuracy of the results are the task for the future.

\section{References}

[1] Colton, D. and Kress, R. (1998). Inverse acoustic and electromagnetic scattering theory, Berlin, Springer.

[2] De Zaeytijd,J., Bogaert,I. and Franchois, A. (2008). An efficient hybrid MLFMA-FFT solver for the volume integral equation in case of sparse 3D inhomogeneous dielectric scatterers, Journal of Computational Physics, 227, 7052-7068.

[3] Yang, J., Abubaker, A., van den Berg, P.M., Habashy, T.M. and Reitich, F. (2008). A CG-FFT approach to the solution of a stress-velocity formulation of three-dimensional elastic scattering problems, Journal of Computational physics, 227, 10018-10039.

[4] Touhei, T. (2009). Generalized Fourier transform and its application to the volume integral equation for elastic wave propagation in a half space, International Journal of Solids and Structures, 46,52-73.

[5] Touhei, T., Takagishi, T. and Wajima, Y. (2009), Analysis of scattered elastic waves in a half space by means of the volume integral equation method. Journal of applied mechanics,(JSCE), 12, 1-8, in Japanese.

[6] Barrett, M., Berry, M., Chan, T.F., Demmel, J., Donato, J. M., Dongarra, J., Eijkhout, V., Pozo, R., Romine, C. and Van der Vorst, H. (1994). Templates for the solution of Linear Systems: Building Blocks for Iterative Methods, SIAM. 